PROCEEDINGS OF THE

AMERICAN MATHEMATICAL SOCIETY

Volume 135, Number 4, April 2007, Pages 1039-1050

S 0002-9939(06)08609-6

Article electronically published on September 18, 2006

\title{
MINIMAL NUMERICAL-RADIUS EXTENSIONS OF OPERATORS
}

\author{
A. G. AKSOY AND B. L. CHALMERS
}

(Communicated by Jonathan M. Borwein)

\begin{abstract}
In this paper we characterize minimal numerical-radius extensions of operators from finite-dimensional subspaces and compare them with minimal operator-norm extensions. We note that in the cases $L^{p}, p=1, \infty$, and in the case of self-adjoint extensions in $L^{2}$, the two extensions and their norms are equal.

We also show that, in the case of $L^{p}, 1<p<\infty$, and more generally in the case of the dual space being strictly convex, if the minimal projections with respect to the operator norm and with respect to the numerical radius have equal norms, then the operator norm is 1 . An analogous result is also true for an arbitrary extension. Finally, we provide an example of a projection from $l_{3}^{p}$ onto a two-dimensional subspace which is minimal with respect to norm but not with respect to the numerical radius for $p \neq 1,2, \infty$, and we determine the minimal numerical-radius projection in this same situation.
\end{abstract}

\section{INTRODUCTION}

A projection from a normed linear space $X$ onto a subspace $V$ is a bounded linear operator $P: X \rightarrow V$ having the property that $\left.P\right|_{V}=I . \quad P$ is called a minimal projection if $\|P\|$ is the least possible. It is known (see [11]) that for a Banach space $X$ and a subspace $V \subset X$, if $V=Z^{*}$ for some Banach space $Z$, then there exists a minimal projection $P: X \rightarrow V$. In this paper we consider projections where the space $V$ is finite-dimensional and thus $\left(V^{*}\right)^{*}=V$.

In general, a given subspace will not be the range of a projection of norm 1, and the projection of least norm is difficult to discover even if its existence is known a priori. A well-known example of the difficult nature of such problems is that the minimal projection of $C[0,1]$ onto the subspace $\pi_{3}$ of polynomials of degree less than or equal to 3 is unknown. For an explicit determination of the projection of the minimal norm from the space $C[-1,1]$ onto $\pi_{2}$, see $[8$

Let $X$ be a Banach space and $V=\left[v_{1}, v_{2}, \ldots, v_{n}\right]$ be a finite-dimensional subspace of $X$. Suppose $T: V \rightarrow V$. We would like to extend $T$ to $\widetilde{T}: X \rightarrow V$ such that the numerical radius $w(\widetilde{T})$ of $\widetilde{T}$ is as small as possible. Notice that when $T=I$, we are considering minimal numerical-radius projections, and if $\operatorname{dim} V=$ 1 , then the extension is the Hahn-Banach extension in the context of numerical radius. The motivation to study extensions or projections with respect to numerical

Received by the editors October 22, 2005.

2000 Mathematics Subject Classification. Primary 41A35; Secondary 41A65.

Key words and phrases. Minimal projections, numerical radius, diagonal extremal pairs.

(C)2006 American Mathematical Society 
radius stems from several factors. First, as we will present in the following section, numerical radius is a "natural" concept in Hilbert spaces, and in Hilbert spaces projections have desirable properties. The second reason is the inequality $w(T) \leq$ $\|T\|$. Therefore, there are more spaces for which $\|T\| \geq 1$ but $w(T)=1$. Moreover, considering extension properties with respect to numerical radius is exactly an $n$ dimensional Hahn-Banach theorem, as illustrated in Example 2.2 below.

In this paper our aim is to characterize minimal numerical-radius extensions of operators from finite-dimensional subspaces and compare them with minimal operator-norm extensions. We note that in the cases $L^{p}, p=1, \infty$, and in the case of self-adjoint extensions in $L^{2}$, the two extensions and their norms are equal. (This result is well known and we provide a simple proof for the sake of completeness.) Consequently, all known theorems for minimal operator-norm extensions in the cases of $L^{1}$ and $L^{\infty}$ also apply to minimal numerical-radius extensions in these settings.

We also show that in the case of $L^{p}, 1<p<\infty$, and more generally in the case of the dual space being strictly convex, if the minimal projection with respect to the operator norm and that with respect to numerical radius have equal norms, then that operator norm is 1 . This result should be viewed together with the result of A. E. Taylor [18, where it is proved that if the unit sphere in the conjugate space is strictly convex, then every continuous linear functional on an arbitrary linear subspace of $X$ has a unique extension without increase of norm to all of $X$. (S. R. Foguel in [13] proves the converse. Namely, if $X^{*}$ is not strictly convex, then there exists a bounded linear functional defined on a linear subspace of $X$ for which the norm-preserving linear extension to $X$ is not unique.)

Finally, we provide an example of a projection from $l_{3}^{p}$ onto a two-dimensional subspace which is minimal with respect to norm, but not with respect to the numerical radius, for $p \neq 1,2, \infty$. We also determine the minimal numerical-radius projection in this same situation. Here, once again, we have uniqueness of the projections. This follows from the result of V.P. Odinec. In [17], he proves that minimal projections of norm greater than one from a three-dimensional Banach space onto any of its two-dimensional subspaces are unique.

\section{CharaCterization of Minimal NUMERICAL-RADiUs EXTEnsions}

In this section we give definitions of basic concepts, which lead us to a characterization (in Theorem 2.1) of minimal numerical-radius extensions. Throughout the paper, $X$ is a Banach space, $X^{*}$ is its dual and the field is the real numbers $\mathbf{R}$ or the complex numbers $\mathbf{C}$. The transformation $T$ taking a finite-dimensional subspace $V=\left[v_{1}, v_{2}, \ldots, v_{n}\right]$ of $X$ into itself is given by

$$
T=\sum_{i=1}^{n} u_{i} \otimes v_{i}: V \rightarrow V=\left[v_{1}, v_{2}, \ldots, v_{n}\right] \subset X, \text { where } u_{i} \in V^{*},
$$

and

$$
\widetilde{T}=\sum_{i=1}^{n} \widetilde{u}_{i} \otimes v_{i}: X \rightarrow V, \text { where } \widetilde{u}_{i} \in X^{*}
$$

Clearly, $\widetilde{T}$ is an extension of $T$ to all of $X$. In this paper we will investigate the conditions, under which $\widetilde{T}$ has as small a (possibly minimal) numerical radius as defined in Definition 2.4 below. 
Following the usual practice, let $B(X)$ and $S(X)$ denote the closed unit ball and unit sphere of $X$, respectively. Let $\mathcal{B}=\mathcal{B}(X, V)$ be the space of all bounded linear operators from $X$ into a finite-dimensional subspace $V$ of $X$. Let $\mathcal{T}$ be the family of all operators in $\mathcal{B}(X, V)$ with a given action on $V$ (e.g., the identity action corresponds to the family of bounded projections onto $V)$. Finally, $\left\langle x, x^{*}\right\rangle$ denotes the action of $x^{*} \in X^{*}$ on $x \in X$.

Before defining an extremal of $x \in X$, we consider the example of the classical Hölder Inequality. For suitable conjugate pairs $p$ and $q$ and (for simplicity) for $f g \neq 0$ a.e., if $X=L^{p}$ and $X^{*}=L^{q}$, then $|\langle f, g\rangle| \leq\|f\|_{p}\|g\|_{q}$ with equality if and only if $\alpha(\operatorname{sgn} f)|f|^{p}=\beta(\operatorname{sgn} g)|g|^{q}$ a.e. for some constants $\alpha, \beta$ with $\alpha \beta>0$. In the case of equality, we say $g$ is an extremal of $f$ and $f$ is an extremal of $g$, and write $g=(\alpha / \beta)^{1 / q}(\operatorname{sgn} f)|f|^{p / q}=$ : ext $f$ or $f=(\beta / \alpha)^{1 / p}(\operatorname{sgn} g)|g|^{q / p}=$ : ext $g$.

Definition 2.1. Let $X$ denote a Banach space. If $x \in X$ and $x^{*} \in X^{*}$ are such that $\left|\left\langle x, x^{*}\right\rangle\right|=\|x\| \cdot\left\|x^{*}\right\| \neq 0$, then $x^{*}$ is an extremal of $x$ and we write $x^{*}=$ ext $x$. Similarly $x$ is an extremal of $x^{*}$, written $x=$ ext $x^{*}$.

Note that ext $x$ multiplied by any nonzero scalar factor is still an extremal of $x$. Note further that, if $X$ is not smooth, for some $x$ there may be many $x^{*}$ (which differ by more than a scalar multiple) that are extremals of $x$. The fact that, for each $x$, there may be more than one extremal and that neither $x$ nor ext $x$ is necessarily normalized is not important for our purposes because the operator $E_{\widetilde{T}}$ (see (11) below) is a positive combination of dyads $(y \otimes e x t y)$.

Definition 2.2. If $T$ is a bounded linear map on $X$, the numerical range $W(T)$ and the numerical radius $w(T)$ are defined by

$$
W(T)=\left\{\left\langle T x, x^{*}\right\rangle:\left(x, x^{*}\right) \in S(X) \times S\left(X^{*}\right),\left\langle x, x^{*}\right\rangle=1\right\}
$$

and

$$
w(T)=\sup \{|\lambda|: \lambda \in W(T)\}
$$

In the above definition of the numerical range the condition $\left\langle x, x^{*}\right\rangle=1$ implies that the points considered are of the type $\left(x, x^{*}\right)=\left(\right.$ ext $\left.x^{*}, x^{*}\right)$.

Moreover, $W(T) \subset\left\{\left\langle T x, x^{*}\right\rangle:\left(x, x^{*}\right) \in B(X) \times B\left(X^{*}\right)\right\}$ and $w(T) \leq\|T\|$. Naturally, for $\|T\|$ we are taking "the supremum" over $\left(x, x^{*}\right) \in B(X) \times B\left(X^{*}\right)$ but for $w(T)$ "the supremum" is taken over those $\left(x, x^{*}\right)$ for which $\left\langle x, x^{*}\right\rangle=1$. Quite often $w$ is actually a norm equivalent to the operator norm $\|\cdot\|$.

These definitions lead us to the notion of the numerical index, $n(X)$, of the space $X$. This constant is defined by $n(X)=\inf \{w(T): T \in \mathcal{B}(X),\|T\|=1\}$. Note that $0 \leq n(X) \leq 1, n(X)>0$ if and only if $w$ and $\|\cdot\|$ are equivalent norms, and $n(X)=1$ if and only if $w(T)=\|T\|$ for all $T \in \mathcal{B}(X)$.

A complete survey of numerical ranges, their close relation to spectral theory and their applications can be found in [2], 10] and [14].

If $T: H \rightarrow H$ is a bounded linear operator on a Hilbert space, then the numerical radius takes the form $w(T)=\sup \{|\langle T x, x\rangle|:\|x\|=1\}$, because for each linear functional $x^{*}$ there is a unique $x_{0} \in H$ such that $x^{*}(x)=\left\langle x, x_{0}\right\rangle$ for all $x \in H$. Moreover, if $T$ is a self-adjoint or a normal operator on a Hilbert space $H$, then $\|T\|=w(T)$. Notice also that if a nonzero $T: H \rightarrow H$ is self-adjoint and compact, then $T$ has an eigenvalue $\lambda$ such that $w(T)=\|T\|=\lambda$. 
These properties of the numerical radius together with the properties of orthogonal projections from Hilbert spaces onto closed subspaces are the motivation to investigate minimal extensions or projections with respect to the numerical radius.

Definition 2.3. We call $(x, y)=($ ext $y, y) \in S\left(X^{* *}\right) \times S\left(X^{*}\right)$ a diagonal extremal pair for $\widetilde{T} \in \mathcal{B}(X, V)$ if $\left\langle\widetilde{T}^{* *} x, y\right\rangle=w(\widetilde{T})$, where $\widetilde{T}^{* *}: X^{* *} \rightarrow V$ is the second adjoint extension for $\widetilde{T}$ and $V=\left[v_{1}, v_{2}, \ldots, v_{n}\right] \subset X$.

To clarify our notation, note that the map $\widetilde{T}$ has the expression

$$
\widetilde{T}=\sum_{i=1}^{n} \widetilde{u}_{i} \otimes v_{i}: X \rightarrow V
$$

and $\widetilde{T} x=\sum_{i=1}^{n}\left\langle x, \widetilde{u}_{i}\right\rangle v_{i}$ with $\widetilde{u}_{i} \in X^{*}, v_{i} \in V$, where $\left\langle x, \widetilde{u}_{i}\right\rangle$ denotes the functional $\widetilde{u}_{i}$, is acting on $x$. The map $\widetilde{T}^{* *}$ can be expressed as

$$
\widetilde{T}^{* *}=\sum_{i=1}^{n} u_{i} \otimes v_{i}: X^{* *} \rightarrow V
$$

and $\widetilde{T}^{* *} x=\sum_{i=1}^{n}\left\langle u_{i}, x\right\rangle v_{i}$ with $u_{i} \in X^{* * *}, v_{i} \in V\left(x \in X^{* *}\right)$ and $\left\langle u_{i}, x\right\rangle$ denotes the action of $x$ on $u_{i}$.

The set of all diagonal extremal pairs will be denoted $\mathcal{E}_{w}(\widetilde{T})$,

$$
\mathcal{E}_{w}(\widetilde{T})=\left\{(\text { ext } y, y) \in S\left(X^{* *}\right) \times S\left(X^{*}\right): \sum_{i=1}^{n}\left\langle\text { ext } y, u_{i}\right\rangle \cdot\left\langle v_{i}, y\right\rangle=w(\widetilde{T})\right\} .
$$

To each $(x, y) \in X^{* *} \times X^{*}$ associate the rank-one operator $y \otimes x: X \rightarrow X^{* *}$ given by

$$
(y \otimes x)(z)=\langle z, y\rangle x
$$

for $z \in X$. Accordingly, to each $(x, y) \in \mathcal{E}_{w}(\widetilde{T})$ associate the rank-one operator $y \otimes$ ext $y: X \rightarrow X^{* *}$ given by

$$
(y \otimes \text { ext } y)(z)=\langle z, y\rangle \text { ext } y
$$

for $z \in X$. The usual definition for the set of all extremal pairs for $\widetilde{T}$, denoted $\mathcal{E}(\widetilde{T})$, is given in [5] as:

$$
\mathcal{E}(\widetilde{T})=\left\{(x, y) \in S\left(X^{* *}\right) \times S\left(X^{*}\right): \sum_{i=1}^{n}\left\langle x, u_{i}\right\rangle \cdot\left\langle v_{i}, y\right\rangle=\|\widetilde{T}\|\right\} .
$$

In the case of diagonal extremal pairs we require $\mid\langle$ ext $y, y\rangle \mid=1$.

Definition 2.4. Let $T=\sum_{i=1}^{n} u_{i} \otimes v_{i}: V \rightarrow V=\left[v_{1}, v_{2}, \ldots, v_{n}\right] \subset X$, where $u_{i} \in V^{*}$ and $X$ is a Banach space. Let $\widetilde{T}=\sum_{i=1}^{n} \widetilde{u}_{i} \otimes v_{i}: X \rightarrow V$ be an extension of $T$ to all of $X$. We say $\widetilde{T}$ is a minimal numerical-radius extension of $T$ if $w(\widetilde{T})=\inf \left\{w(S): S: X \rightarrow V\right.$ and $\left.\left.S\right|_{V}=T\right\}$. Clearly $w(T) \leq w(\widetilde{T})$.

Theorem 2.1 (Characterization). $\widetilde{T}$ is a minimal numerical-radius extension of $T$ (if $T=I, \widetilde{T}=P$ is a minimal numerical-radius projection from $X$ onto $V$ ) if and only if the closed convex hull of $\{y \otimes x\}$, where $(x, y) \in \mathcal{E}_{w}(\widetilde{T})$, contains an operator for which $V$ is an invariant subspace. 
Proof. One can appropriately modify the proof given in [5], Theorem 1, as follows. The problem is equivalent to best approximating, in the numerical-radius norm, a fixed operator $T_{0} \in \mathcal{T}$ from the space of operators $\mathcal{D}=\{\Delta \in \mathcal{B}: \Delta=0$ on $V\}=$ $\operatorname{sp}\left\{\delta \otimes v: \delta \in V^{\perp}, v \in V\right\}$. Let $K=B\left(X^{* *}\right) \times B\left(X^{*}\right)$ endowed with the product topology, where $B(\cdot *)$ denotes the unit ball with its weak* topology. Then $K$ is compact. Let

$$
K_{w}=K \cap \operatorname{Diag}=\left\{(x, y) \in B\left(X^{* *}\right) \times B\left(X^{*}\right): x=\operatorname{ext}(y) \text { or } x=0\right\} .
$$

Then $K_{w}$ is compact since $K \cap$ Diag is a closed subset of $K$, as can be seen from the following argument. Take $\left\{\left(x_{i}, y_{i}\right)\right\}_{i \in I}$ a net in $K_{w}$ and $(x, y) \in X^{* *} \times X^{*}$ such that $\left(x_{i}, y_{i}\right)$ converges weak ${ }^{*}$ to $(x, y)$. Clearly $(x, y) \in B\left(X^{* *}\right) \times B\left(X^{*}\right)$. Now consider two cases:

1) If $x=0$, we are done since $(0, y) \in K_{w}$.

2) If $x \neq 0$, we have to prove that $x=\operatorname{ext}(y)$, that is, $|x(y)|=\|x\|\|y\|$. We have

$$
\|x\| \leq \liminf _{i}\left\|x_{i}\right\| \text { and }\|y\| \leq \liminf _{i}\left\|y_{i}\right\|
$$

therefore

$$
\|x\|\|y\| \leq \lim _{k}\left\|x_{i_{k}}\right\|\left\|y_{i_{k}}\right\|=\lim _{k}\left|x_{i_{k}}\left(y_{i_{k}}\right)\right| .
$$

Setting

$$
x_{i_{k}}\left(y_{i_{k}}\right)=x_{i_{k}}\left(y_{i_{k}}-y\right)+\left(x_{i_{k}}-x\right)(y)+x(y)
$$

and replacing $X^{*}$ by $V^{*}$, we obtain that $\left\{y_{i}\right\}$ is strongly convergent to $y$, which implies $\|x\|\|y\| \leq|x(y)|$, which obviously leads to $\|x\|\|y\|=|x(y)|$. This shows that $K_{w}$ is closed.

Thus the set $\mathcal{E}(\widetilde{T})$, being the set of points where a continuous (bilinear) function achieves its maximum on a compact set, is not empty.

Associate with any operator $Q \in \mathcal{B}$ the bilinear form $\widehat{Q} \in C\left(K_{w}\right)$ via $\widehat{Q}(x, y)=$ $\left\langle Q^{* *} x, y\right\rangle$, and let $\widehat{\mathcal{D}}=\{\widehat{\Delta}: \Delta \in \mathcal{D}\}$. Then, making use of standard duality theory for $C\left(K_{w}\right), K_{w}$ compact (see e.g., [15], Theorem 1.1 (p.18) and Theorem $1.3(\mathrm{p} .29)$ ), we have that $\widehat{T}=\widehat{T}_{0}-\widehat{\Delta}_{0}$ is of minimal norm if and only if there exists a finite, nonzero (total mass one) signed measure $\hat{\mu}$ supported on the critical set

$$
\mathcal{C}_{w}(\widehat{T}):=\left\{(x, y) \in\left(S\left(X^{* *}\right) \times S\left(X^{*}\right)\right) \cap \operatorname{Diag}:|\widehat{T}(x, y)|=w(\widetilde{T})\right\}
$$

such that $\operatorname{sgn} \hat{\mu}\{(x, y)\}=\operatorname{sgn} \widehat{T}(x, y)$ and $\hat{\mu} \in \widehat{\mathcal{D}}^{\perp}$, i.e.,

$$
0=\int_{\mathcal{C}_{w}(\widehat{T})} \widehat{\Delta} d \hat{\mu} \quad \text { for all } \widehat{\Delta} \in \widehat{\mathcal{D}} .
$$

But now, since any $\widehat{Q} \in\{\widehat{T}\} \cup \widehat{\mathcal{D}}$ is a bilinear function, we can replace the signed measure $\hat{\mu}$, supported in $\mathcal{C}_{w}(\widehat{T})$, by a positive measure $\mu$ supported on $\mathcal{E}_{w}(\widetilde{T}) \subset$ $\mathcal{C}_{w}(\widehat{T})$ by noting that

$$
\mathcal{C}_{w}(\widehat{T})=\left\{\left(x, e^{i \theta} y\right):(x, y) \in \mathcal{E}_{w}(\widetilde{T}) \text { and } \theta \in I\right\},
$$

where $I=[0,2 \pi)$ in the complex case and $I=\{0, \pi\}$ in the real case, and setting

$$
\mu\{(x, y)\}=|\hat{\mu}|\left\{\left(x, e^{i \theta} y\right): \theta \in I\right\} .
$$

Then $\operatorname{sgn} \mu\{(x, y)\}=\operatorname{sgn} \widehat{T}(x, y)=1$ for $(x, y) \in \mathcal{E}_{w}(T)$, and

$$
0=\int_{\mathcal{E}_{w}(\widetilde{T})} \widehat{\Delta} d \mu \text { for all } \Delta \in \mathcal{D},
$$


since

$$
\begin{aligned}
\int_{\mathcal{C}_{w}(\widehat{T})} \widehat{\Delta} d \hat{\mu} & =\int_{\substack{(x, y) \in \mathcal{E}_{w}(\widetilde{T}) \\
\theta \in I}} \widehat{\Delta}\left(x, e^{i \theta} y\right) d \hat{\mu}\left(x, e^{i \theta} y\right) \\
& =\int_{\substack{(x, y) \in \mathcal{E}_{w}(\widetilde{T}) \\
\theta \in I}} e^{-i \theta} \widehat{\Delta}(x, y) e^{i \theta} d|\hat{\mu}|\left(x, e^{i \theta} y\right)=\int_{\mathcal{E}_{w}(\widetilde{T})} \widehat{\Delta} d \mu .
\end{aligned}
$$

Hence,

$$
\begin{aligned}
0=\int_{\mathcal{E}_{w}(\widetilde{T})} \widehat{\Delta} d \mu & =\int_{\mathcal{E}_{w}(\widetilde{T})}\left\langle\Delta^{* *} x, y\right\rangle d \mu(x, y) \\
& =\int_{\mathcal{E}_{w}(\widetilde{T})}\langle x, \delta\rangle\langle v, y\rangle d \mu(x, y)=\left\langle\int_{\mathcal{E}_{w}(\widetilde{T})}\langle v, y\rangle x d \mu(x, y), \delta h\right\rangle
\end{aligned}
$$

for all $\Delta=\delta \otimes v\left(\delta \in V^{\perp}, v \in V\right)$, where, for $z \in X, \int_{\mathcal{E}_{w}(\widetilde{T})}\langle z, y\rangle x$

$\left\langle d \mu(x, y)\right.$ is the element $w \in X^{* *}$ defined by $\left\langle x^{*}, w\right\rangle=\int_{\mathcal{E}_{w}(\widetilde{T})}\langle z, y\rangle\left\langle x^{*}, x\right\rangle d \mu(x, y)$ for all $x^{*} \in X^{*}$. $\widetilde{T}$ is minimal, therefore, if and only if $\int_{\mathcal{E}_{w}(\widetilde{T})}\langle v, y\rangle x d \mu(x, y) \in$ $\left(V^{\perp}\right)^{\perp}=V$, i.e., if and only if there exists an operator (from $X$ into $X^{* *}$ )

$$
E_{\widetilde{T}}=\int_{\mathcal{E}_{w}(\widetilde{T})} y \otimes x d \mu(x, y): \quad V \rightarrow V .
$$

The proof of the theorem is complete.

Remark 2.1. The existence of a minimal projection as characterized in Theorem 2.1 follows from [11] and the fact that $V$ is a finite-dimensional subspace of $X$. In the proof of Theorem 2.1 we used Singer's identification (see [15, Theorem 1.1 (p.18) and Theorem 1.3 (p.29)) of finding a minimal operator as the error of best approximation in $C(K)$ for $K$ compact. It is possible to use other methods, such as the Kolmogorov criterion for best approximation (see [15], Theorem 1.16 (p.69)).

Remark 2.2. We can restate Theorem 2.1 as follows:

$\widetilde{T}$ is a minimal numerical radius extension of $T$ if and only if there exists a probability measure $\mu$ on $\mathcal{E}_{w}(\widetilde{T})$ such that for $(x, y) \in \mathcal{E}_{w}(\widetilde{T})$, the operator $E_{\widetilde{T}}$ : $X \rightarrow X^{* *}$ defined by

$$
E_{\widetilde{T}}:=\int y \otimes x d \mu(x, y)
$$

maps $V$ into $V$.

Moreover, by taking a fixed basis $\vec{v}=\left(v_{1}, v_{2}, \ldots, v_{n}\right)$ for $V$, one can write (11) as the system of $n$ equations

$$
\int\langle\vec{v}, y\rangle x d \mu(x, y)=M \vec{v}
$$

for some matrix $M$.

Notice that the family $\mathcal{T}$ of operators in $\mathcal{B}(X, V)$ with a given action on $V$ can be expressed as

$$
\mathcal{T}=\left\{\sum_{i=1}^{n} u_{i} \otimes v_{i}:\left\langle v_{i}, u_{j}\right\rangle=A_{i j} \text { for } A \text { a given fixed } n \times n \text { matrix }\right\} .
$$


The matrix $M$ in equation (2) may be regarded as a function of this matrix $A$. Hence, equation (2) can determine a minimal numerical radius of $\widetilde{T}$, up to $n^{2}$ entries of $M$.

The following elementary examples illustrate Theorem 2.1 and tell us what to take as the map $E_{\widetilde{T}}$.

Example 2.1 (Hilbert space case). Let $X$ be a Hilbert space and let $\vec{v}=$ $\left\{v_{1}, v_{2}, \ldots, v_{n}\right\}$ be an orthonormal basis for $V$. Let $\mathcal{T}$ have a fixed "diagonal" action, i.e., $A_{i j}=d_{i} \delta_{i j}$, where $d=\left(d_{1}, d_{2}, \ldots, d_{n}\right)$ is a fixed $n$-tuple of scalars, and let $\lambda=\max _{i}\left|d_{i}\right|=\left|d_{i_{0}}\right|$.

Then $\widetilde{T}=\sum_{i=1}^{n} d_{i} v_{i} \otimes v_{i}$ has a minimal numerical-radius because we can find diagonal extremal pairs of the form $(\sigma z, z)$ with $z$ an arbitrary norm-1 element of the eigenspace corresponding to a maximum eigenvalue $\left|d_{i_{0}}\right|$ and $\sigma=\operatorname{sgn} d_{i_{0}}$. (Note that $\|\widetilde{T}\|=\lambda$. Then $\langle\widetilde{T} \sigma z, z\rangle=\lambda$. Thus $E_{\widetilde{T}}=z \otimes \sigma z$ for any choice of $z$ described above.)

Example 2.2. The following example provides us with another reason to study minimal extensions with respect to the numerical radius. It turns out that for the case $n=1(\operatorname{dim} V=1)$ the minimal numerical-radius extension corresponds to the classical Hahn-Banach extension. To see this, consider a rank-one projection $P=u \otimes v$, such that

$$
\|P\|=\sup _{\|x\|=\|y\|=1}\langle x, u\rangle \cdot\langle v, y\rangle=\|u\| \cdot\|v\| \geq\langle u, v\rangle=1 .
$$

Since $P$ is a projection, $1 \leq w(P) \leq\|P\|$, but in the minimal case we have the equality $\|P\|=w(P)$ because $\|P\|=1$. For this reason, studying minimal $w(P)$ for higher dimensions can also be viewed as an $n$-dimensional Hahn-Banach theorem.

\section{Diagonal extremal pairs and minimal projections in $L^{p}, p=1,2, \infty$}

Throughout the remainder of this paper, in the case of $L^{1}$, it will be assumed that $(\Lambda, \Sigma, \mu)$ is a measure space for which $\left(L^{1}\right)^{*}=L^{\infty}$. For example, $\left(L^{1}\right)^{*}=L^{\infty}$ if the measure space $(\Lambda, \Sigma, \mu)$ is $\sigma$-finite. We write $L^{1}=L^{1}(\Lambda, \Sigma, \mu)$ and $L^{p}=L^{p}(\Lambda, \Sigma, \mu)$.

The following result is well known and follows from the fact (see [2], §9, and [12]) that $n\left(L^{p}\right)=1$ for $p=1, \infty$, and the fact that self-adjoint operators have numericalradius equal to the operator-norm. We state this fact as a theorem (and include a proof for the sake of completeness), so that we can mention several corollaries, which are the results of recent work, and so that we can apply this theorem in Example 4.2 after Theorem 4.1 below.

Theorem 3.1 (When extensions coincide). In the cases $X=L^{p}, p=1, \infty$, the minimal numerical-radius extensions and the minimal operator-norm extensions coincide, with the same norms. Also in the case of self-adjoint extensions in $L^{2}$, the two extensions (with respect to norm and with respect to numerical radius) and their respective norms are equal.

Proof. In the case $p=1$, it is well known that $\|\widetilde{T}\|=\sup _{t \in \Lambda} L(t)$, where $L(t)$ is the so-called Lebesgue function of $\widetilde{T}$, defined as

$$
L(t)=\int_{\Lambda}\left|\sum_{i=1}^{n} u_{i}(t) v_{i}(s)\right| d \mu(s)=\left\|\sum_{i=1}^{n} u_{i}(t) v_{i}\right\|_{L^{1}} .
$$


Similarly, in the case $p=\infty,\|\widetilde{T}\|=\sup _{t \in \Lambda} L(t)$, where $L(t)$ is the Lebesgue function of $\widetilde{T}$, defined as

$$
L(t)=\int_{\Lambda}\left|\sum_{i=1}^{n} u_{i}(s) v_{i}(t)\right| d \mu(s)=\left\|\sum_{i=1}^{n} v_{i}(t) u_{i}\right\|_{L^{1}} .
$$

Thus in both cases we see that the extremal pairs (used in minimal operator extensions) are of the form (ext $y, y)$, where $y \in B\left(X^{*}\right)$. In fact in the case $L^{1}$, $($ ext $y, y)=\left(\delta_{t}, \operatorname{sgn}(\vec{u}(t) \cdot \vec{v})\right)$ and in the case $L^{\infty},($ ext $y, y)=\left(\operatorname{sgn}(\vec{u} \cdot \vec{v}(t)), \delta_{t}\right)$, where $\delta_{t}$ denotes the usual "delta" function at $t$. The set of all diagonal critical pairs will be denoted $\mathcal{C}_{w}(\widetilde{T})$,

$$
\begin{aligned}
\mathcal{C}_{w}(\widetilde{T})=\left\{(\text { ext } y, y) \in S\left(X^{* *}\right) \times S\left(X^{*}\right):\right. \\
\left.\sum_{i=1}^{n}\left\langle\operatorname{ext} y, u_{i}\right\rangle \cdot\left\langle v_{i}, y\right\rangle=\sigma w(\widetilde{T}),|\sigma|=1\right\} .
\end{aligned}
$$

The set of all diagonal pairs will be $\operatorname{denoted} \mathcal{C}(\widetilde{T})$,

$$
\begin{aligned}
& \mathcal{C}(\widetilde{T})=\left\{(\text { ext } y, y) \in S\left(X^{* *}\right) \times S\left(X^{*}\right):\right. \\
& \left.\qquad \sum_{i=1}^{n}\left\langle\text { ext } y, u_{i}\right\rangle \cdot\left\langle v_{i}, y\right\rangle=\sigma\|\widetilde{T}\|,|\sigma|=1\right\} .
\end{aligned}
$$

Thus, in the cases $p=1, \infty, \mathcal{C}(\widetilde{T}) \subset \mathcal{C}_{w}(\widetilde{T})$, but since $w(\widetilde{T}) \leq\|\widetilde{T}\|$ in these cases, the conclusion follows immediately.

In the case $p=2$, for any self-adjoint operator, we have $\|\widetilde{T}\|=w(\widetilde{T})=|\lambda|$, where $\lambda$ is the maximum (in modulus) eigenvalue. Indeed in this case $\|\widetilde{T}\|=w(\widetilde{T})=$ $|\langle\widetilde{T} x, x\rangle|$, where $x$ is a norm-1 "maximum" eigenvector. The proof of the theorem is complete.

Corollary 3.1 (Maximal Spaces). The maximal spaces with respect to the numerical radius coincide with the maximal spaces with respect to the operator norm. Here by "maximal space with respect to the numerical radius" we mean an $n$-dimensional space $V$ yielding $\sup w(\widetilde{T})$, where $\widetilde{T}$ is given in Definition 2.4 with $T=I$. "Maximal space with respect to the operator norm" is defined similarly.

Proof. It is well known (e.g., see [19]) that $L^{\infty}$ is a "maximal overspace" for any finite-dimensional Banach space. Since in the case $L^{\infty}$, however, the minimal numerical-radius extensions and the minimal operator-norm extensions coincide, the result follows. (It is also known that isometric copies of the maximal symmetric spaces lie in $L^{1}$ (see [4) and that isometric copies of the maximal (unrestricted) spaces lie in $L^{1}$ (see [16]).)

Corollary 3.2. Let $T=\sum_{i=1}^{2} u_{i} \otimes v_{i}: V \rightarrow V=\left[v_{1}, v_{2}\right] \subset X$, where $u_{i} \in V^{*}$ and $X$ is a Banach space. Let $\widetilde{T}=\sum_{i=1}^{2} \widetilde{u}_{i} \otimes v_{i}: X \rightarrow V$ be a minimal numericalradius extension of $T$ to all of $X$. When the field is $\mathbb{R}$ there exists a rank-2 $T$ such that $w(T)=w(\widetilde{T})$ for all $X$ if and only if the unit ball of $V$ is either not smooth or not strictly convex. 
Proof. The result follows from the fact that any such space is isometric to a subspace of $L^{1}$, from the fact that $L^{1}$ is a "maximal overspace" for any such space, from Theorem 3.1, and from [9].

Remark 3.1. In the case of $X=L^{1}$, just as in [6], there is a simple geometric description for $T$.

Remark 3.2. Note that the condition of self-adjointness is critical in the $L^{2}$ case of the above theorem. If, for example, the field is $\mathbb{R}$ and $T: \ell_{2}^{2} \rightarrow \ell_{2}^{2}$ is given by $T\left(x_{1}, x_{2}\right)=\left(-x_{2}, x_{1}\right)$, then $w(T)=\sup _{\|x\|=1}\langle T x, x\rangle=0$, while $\|T\|=1$. Thus, if the field is $\mathbb{R}$, then $n\left(\ell_{2}^{2}\right)=0$. However, note that if the field is $\mathbb{C}$, then $w(T)=\|T\|=1$. Indeed, if the field is $\mathbb{C}$, then in all cases $w(T) \leq\|T\| \leq 2 w(T)$ (e.g., see [10]).

\section{Minimal projections with Respect to the numerical Radius in $L^{p}$}

Following is an example of a particular projection which remains minimal with respect to norm and with respect to numerical radius simultaneously. Similar projections are considered in [1].

Example 4.1. Consider the Rademacher functions $r_{i}(t)=(-1)^{\left[2^{i} t\right]}$, for $0 \leq t \leq$ $1, i=1,2, \ldots, n$. For $\vec{r}=\left(r_{1}, r_{2}, \ldots, r_{n}\right)$, let $[\vec{r}]$ be the space spanned by the Rademacher functions. It can be seen from the Khintchine inequality that $\ell_{2}^{n} \approx$ $[\vec{r}] \subset L^{p}[0,1]$ for all $1 \leq p \leq \infty$.

Let $P: L^{p}[0,1] \rightarrow[\vec{r}]$ be the Rademacher projection given as $P=\sum_{i=1}^{n} r_{i} \otimes r_{i}$. In [7, Theorem 3, it is proved that for $n=2,\|P\|=1$.

This result together with the inequality $w(P) \leq\|P\|$ shows that the projection $P: L^{p}[0,1] \rightarrow[\vec{r}]$ with $\vec{r}=\left(r_{1}, r_{2}\right)$ is minimal with respect to operator norms as well as with respect to numerical radius.

Notation 4.1. Let $P$ denote a minimal projection with respect to the operator norm and let $P_{w}$ denote a minimal projection with respect to the numerical radius.

Theorem 4.1 (Strictly convex spaces). Let $X^{*}$ be strictly convex. Then $\|P\|=$ $w\left(P_{w}\right)$ implies that $\|P\|=1$.

Proof. Let $P=\sum_{i=1}^{n} u_{i} \otimes v_{i}$. Note that

$$
\|P\| \geq w(P) \geq w\left(P_{w}\right)=\|P\|
$$

and hence $\|P\|=w(P)$. Let $(x, y)=($ ext $y, y) \in \mathcal{E}(P)$. Next, letting $\vec{u}=$ $\left(u_{1}, \cdots, u_{n}\right)$, we have

$$
\langle x, \vec{u}\rangle \cdot\langle\vec{v}, y\rangle=\langle x,\langle\vec{v}, y\rangle \cdot \vec{u}\rangle=\|P\|
$$

and thus $x=\operatorname{ext}\langle\vec{v}, y\rangle \cdot \vec{u}=\operatorname{ext} y$. But since $X^{*}$ is strictly convex, we have also that $\langle\vec{v}, y\rangle \cdot \vec{u}=\lambda y$ for some scalar $\lambda$, i.e., $P^{*} y=\lambda y$, since $P^{*}=\sum_{i=1}^{n} v_{i} \otimes u_{i}$. Furthermore $P^{*}$ is also a projection, whence $\left(P^{*}\right)^{2}=P^{*}$, and thus $\lambda=1$ or $\lambda=0$. But $(x, y)=($ ext $y, y) \in \mathcal{E}(P)$ implies that $(y, x)=(y$, ext $y) \in \mathcal{E}\left(P^{*}\right)$. Thus $\lambda=1$ and so $P^{*} y=y$ implies that

$$
1=\|y\|=\left\|P^{*} y\right\|=\left\|P^{*}\right\|=\|P\| .
$$


Example 4.2. $(\|P\|>1)$ Consider a projection $P: \ell_{3}^{p} \rightarrow V=\left[v_{1}, v_{2}\right]$ where $v_{1}=(1,1,1), v_{2}=(-1,0,1)$, which is minimal with respect to norm. Then $P$ is not minimal with respect to the numerical radius for $p \neq 1,2, \infty$. In fact, we can determine the projection $P_{w}$ which is minimal with respect to the numerical radius and note that $w\left(P_{w}\right) \neq\|P\|$ as follows.

Observe that $V=\left[v_{1}=(1,1,1), v_{2}=(-1,0,1)\right]$ is a two-dimensional subspace of $\ell_{3}^{p}$. Since any projection is given by $u_{1} \otimes v_{1}+u_{2} \otimes v_{2}$, it is easy to see that $u_{1}=\left(\frac{1-d}{2}, d, \frac{1-d}{2}\right)$ and $u_{2}=\left(-\frac{1}{2}, 0, \frac{1}{2}\right)$, so that $P$ and $P_{w}$ are determined up to the one parameter $d$.

The generalized Lebesgue function for $P$ can be expressed as

$$
L(\vec{e})=\langle x, \vec{u}\rangle \cdot\langle\vec{v}, y\rangle=\|\langle x, \vec{u}\rangle \cdot \vec{v}\|_{p},
$$

where

$$
\vec{e}=\langle\vec{v}, y\rangle, x=\operatorname{ext}(\vec{e} \cdot \vec{u}) \text { and }\|P\|=\sup _{\vec{e} \in R^{2}} L(\vec{e}) .
$$

Note that $L(\vec{e})=\|\langle x, \vec{u}\rangle \cdot \vec{v}\|_{p}$ is homogeneous with respect to $\vec{e}$.

Note also that $\|\vec{a} \cdot \vec{v}\|_{p}=\left(\left|a_{1}+a_{2}\right|^{p}+\left|a_{1}\right|^{p}+\left|a_{1}-a_{2}\right|^{p}\right)^{\frac{1}{p}}$.

In the case of the numerical radius, i.e., in the case of $P_{w}$, observe that we have the following equations:

$$
\begin{aligned}
& \frac{\left\langle v_{2}, y\right\rangle}{\left\langle v_{1}, y\right\rangle}=e \text { and therefore }-y_{1}+y_{3}=e\left(y_{1}+y_{2}+y_{3}\right) ; \\
& \|y\|_{q}=1 \text { gives a second equation }\left|y_{1}\right|^{q}+\left|y_{2}\right|^{q}+\left|y_{3}\right|^{q}=1 .
\end{aligned}
$$

Thus, in the case of the numerical radius, we need to modify the Lebesgue function as follows:

$$
L(e)=\sup _{K}\langle e x t y, \vec{u}\rangle \cdot\langle\vec{v}, y\rangle
$$

where $K=\left\{y_{3}:\left\langle v_{2}, y\right\rangle=e\left\langle v_{1}, y\right\rangle\right.$ and $\left.\|y\|_{q}=1\right\}$.

In both cases, by use of symmetry, we observe that $L(e)=L(-e)$ and then solve the following two equations for $d$ and $e$ :

$$
\begin{gathered}
L(e)=L(0), \\
L^{\prime}(e)=0 .
\end{gathered}
$$

It can then be determined numerically that for

$$
p=\frac{4}{3}, \quad q=\frac{p}{p-1}=4
$$

(whence $\operatorname{ext}(\vec{e} \cdot \vec{u})=\left(u_{1}+e u_{2}\right)^{3}$ ),

$$
d=0.420942 \ldots, \quad e=1.70761 \ldots,
$$

resulting in (see also [3])

$$
\|P\|=1.05251 \ldots .
$$

By Theorem 4.1 we already know $w\left(P_{w}\right) \neq\|P\|$, and setting

$$
d=0.422971 \ldots, \quad e=1.71662 \ldots,
$$

actually results in

$$
w\left(P_{w}\right)=1.02751 \ldots
$$

and it is the minimal possible (see the following remark). 
Remark 4.1. 1) It is seen that, on $\bar{R}, L(\cdot)$ is strictly dominated by its values at 0 and $e$ and $-e$.

Furthermore, an examination of (2) of Section 2 shows that $\mu$ must be supported on at least 3 points $\left[3\right.$. It thus follows that $P$ and $P_{w}$ are indeed minimal.

2) Here once again, we have uniqueness of the projections. This follows from the result of V.P. Odinec. In [17, he proves that minimal projections of norm greater than one from a three-dimensional Banach space onto any of its two-dimensional subspaces are unique.

3) For general $p$, ext $\vec{e} \cdot \vec{u}=\operatorname{sgn}(\vec{e} \cdot \vec{u})|\vec{e} \cdot \vec{u}|^{\frac{q}{p}}$ and so $p=\frac{4}{3}$ yields the especially simple case ext $\vec{e} \cdot \vec{u}=(\vec{e} \cdot \vec{u})^{3}$.

4) Similar reasoning and numerical results can be obtained in the general case $1<p<\infty, p \neq 2$, and, as suggested by Theorem 3.1] $w\left(P_{w}\right) /\|P\|$ approaches 1 as $p$ approaches 1 or 2 or $\infty$.

Notation 4.2. Let $\widetilde{T}$ denote a minimal extension of $T$ with respect to the operator norm and let $\widetilde{T}_{w}$ denote a minimal extension with respect to the numerical radius.

Finally, it is easy to see that Theorem 4.1 and its proof extend as follows:

Theorem 4.2 (Strictly convex spaces). Let $X^{*}$ be strictly convex. Then $\|\widetilde{T}\|=$ $w\left(\widetilde{T}_{w}\right)$ implies that $\|T\|=\|\widetilde{T}\|$.

Remark 4.2. For the case $X=L^{p}, 1<p<\infty, p \neq 2$, there is no analogue to the linear $(*$ )-equation (for $n>1$ ), as on p. 41 of [5], which holds for minimal operatornorm extensions. This fact reflects the quadratic nature of the form of the minimal numerical-radius extension. (In other words, the "diagonal" extremal pairs are of the form $(e x t y, y)$ and not $(x, y)$ where $x$ and $y$ are essentially "independent".) Thus the form

$$
\left\langle P^{* *} x, y\right\rangle=\langle x, \vec{u}\rangle \cdot\langle\vec{v}, y\rangle
$$

is bi-linear, while the form

$$
\left\langle P^{* *} \operatorname{ext} y, y\right\rangle=\langle\operatorname{ext} y, \vec{u}\rangle \cdot\langle\vec{v}, y\rangle
$$

is quadratic in nature.

This essential aspect is also reflected in the difference of complexity between the minimal operator-norm extension and the minimal numerical-radius extension in the example after Theorem 4.1 above.

Remark 4.3. It follows from Theorem 4.1 that, if $X^{*}$ is strictly convex and if $\|P\| \neq 1$, then $\|P\| \neq w\left(P_{w}\right)$. Notice that in this theorem we are comparing the norm and numerical radius of two kinds of minimal extensions $P$ and $P_{w}$ for which $w\left(P_{w}\right)=\|P\|$. Earlier, in Theorem 3.1, we showed that, in the cases $X=L^{p}, p=$ $1, \infty$, minimal numerical-radius extensions and minimal operator-norm extensions coincide. Several questions arise from these theorems.

1) Consider one of these extensions, say $P$, and suppose $w\left(P_{w}\right)=\|P\|$ where $\|P\|$ is minimal. Does it follow that $w(P)$ is minimal too?

2) Lindenstrauss and Tzafriri's characterization of Hilbert spaces by the uniform boundedness of finite-rank projection constants suggests that a strengthened version of Theorem 4.1 might be possible. Namely, if $X^{*}$ is strictly convex and $X$ is not a Hilbert space, is it true that there are many (at least one) finite-dimensional subspaces for which $\|P\| \neq w\left(P_{w}\right)$ ? 


\section{REFERENCES}

[1] A. G. Aksoy, Q-compact sets and Q-compact maps, Math. Japon. 36(1991) 1-7. MR1093346 (92c:47021)

[2] F. F. Bonsall and J. Duncan, Numerical ranges of operators on normed spaces and of elements of normed algebras, London Mathematical Society Lecture Notes Series, No. 2, Cambridge University Press, London, 1971. MR0288583 (44:5779)

[3] B. L. Chalmers, Minimal projections in $l_{3}^{p}$, in preparation.

[4] B. L. Chalmers and G. Lewicki, Symmetric spaces with maximal projection constants, J. Funct. Anal. 200(2003) 1-22. MR1974085 (2004b:46009)

[5] B. L. Chalmers and F. T. Metcalf, A characterization and equations for minimal projections and extensions, J. Operator Theory 32(1994) 31-46. MR1332442 (96c:46014)

[6] B. L. Chalmers and F. T. Metcalf, The determination of minimal projections and extensions in $L^{1}$, Trans. Amer. Math. Soc. 329(1992) 289-305. MR1034660 (92e:41017)

[7] B. L. Chalmers and F. T. Metcalf, Critical points of the $n$-dimensional Khintchine ratio and Rademacher projection norms, Approximation Theory VI Vol. I(1989) 125-128. MR.1090980 (91m:41056)

[8] B. L. Chalmers and F. T. Metcalf, Determination of a minimal projection from $C[1-, 1]$ onto the quadratics, Numer. Funct. Anal. Optim. 11(1990) 1-10. MR1058773 (91d:46027)

[9] B.L. Chalmers and B. Shekhtman, A two-dimensional Hahn-Banach theorem, Proc. Amer. Math. Soc. 129(2001) 719-724. MR1801997 (2001j:46012)

[10] K. E. Gustafson and D. K. M. Rao, Numerical range - The field of values of linear operators and matrices, Springer-Verlag UTX, New York, 1997. MR.1417493 (98b:47008)

[11] E. W. Cheney and P.D. Morris, On the existence and characterization of minimal projections, J. Reine Angew. Math. 279(1974) 61-76. MR0358188 (50:10653)

[12] E. Ed-Dari and M. A. Khamsi, The numerical index of the $L_{p}$ space, Proc. Amer. Math. Soc. 134 (2006) 2019-2025. MR.2215771

[13] S.R. Foguel, On a theorem by A.E. Taylor, Proc. Amer. Math. Soc. 9(1958) 325. MR0093696 $(20: 219)$

[14] G. López, M. Martín and R. Payá, Real Banach spaces with numerical index 1, Bull. London Math. Soc. 31(1999) 207-212. MR1664125 (99k:46024)

[15] I. Singer, Best approximation in normed linear spaces by elements of linear subspaces, Springer-Verlag, Berlin, 1970. MR0270044 (42:4937)

[16] H. König and N. Tomczak-Jaegermann, Finite-dimensional spaces with maximal projection constant lie in $L^{1}$, Studia Math., to appear.

[17] V.P. Odinec, On uniqueness of minimal projections in Banach space, Dokl. Akad. Nauk SSSR 220(1975) 779-781 (in Russian). MR0470666 (57:10412)

[18] A. E. Taylor, The extension of linear functionals, Duke Math. J. 5(1939) 538-547. MR0000345 (1:58b)

[19] N. Tomczak-Jaegermann, Banach-Mazur distances and finite-dimensional operator ideals, John Wiley and Sons, New York (1989). MR0993774 (90k:46039)

Department of Mathematics, Claremont McKenna College, Claremont, California 91711

E-mail address: asuman.aksoy@claremontmckenna.edu

Department of Mathematics, University of California, Riverside, California 92507

E-mail address: blc@math.ucr.edu 